Proyecciones Journal of Mathematics

Vol. 40, $\mathrm{N}^{o}$ 5, pp. 1137-1145, October 2021.

Universidad Católica del Norte

Antofagasta - Chile

\title{
Sequence spaces defined via Euler method and matrix transformations
}

\author{
Pranav Sharma \\ IIMT University, India \\ Received : October 2020. Accepted : February 2021
}

\begin{abstract}
A blend of matrix summability and Euler summability transformation methods is used to define Lacunary sequence spaces defined over $n$-normed space. Then we present the properties of this space and finally, some inclusion relations are presented.
\end{abstract}

Subjclass [2020]: Primary 40J05; Secondary 46445.

Keywords: Euler summability; n-normed spaces; Orlicz function. 


\section{Introduction}

Accelerated convergence techniques are useful in computer science especially in making graphics and to find eigenvalues and eigenvectors of dynamical systems. Euler summability plays a prominent role in improving the convergence of a given series. For a positive real $q$ and a non negative integer $n$, the Euler transform (see. [1]) $E_{n}^{q}$ of sequence $S=\left(s_{n}\right)$ of the partial sum of a series $\sum_{k=0}^{\infty} a_{k}$ is defined as $E_{n}^{q}(S)=\frac{1}{(1+q)^{n}} \sum_{v=0}^{n}\left(\begin{array}{c}n \\ v\end{array}\right) q^{n-v} s_{v}$. A series $\sum a_{n}$ is said to be $E_{n}^{q}$-summable to $s$ if $E_{n}^{q}(S) \rightarrow s$, as $n \rightarrow \infty$, further, if $\sum_{k}\left|E_{k}^{q}(S)-E_{k-1}^{q}(S)\right|<\infty$ then the sequence is said to be absolutely $E_{n}^{q}$-summable. Let $x=\left(x_{n}\right)$ be a sequence of scalars, for $n \geq 1$ we will denote by $N_{n}(x)$ the difference $E_{n}^{q}(x)-E_{n-1}^{q}(x)$, where $E_{n}^{q}$ is defined as above.

Another important transform of numerical analysis is "Abel's transform" which is defined as $A_{k}=\sum_{j=0}^{k}\left[\frac{q}{1+q}\left(\begin{array}{c}n \\ j\end{array}\right)-\left(\begin{array}{c}n-1 \\ j\end{array}\right)\right] q^{n-(j+1)}$.

Using Abel's transform we have

$$
N_{n}(x)=-\frac{1}{(1+q)^{n-1}}\left(\sum_{j=0}^{n-2} x_{j+1} A_{j}+s_{n-1} A_{n-1}\right)+\frac{1}{(1+q)^{n}}\left(s_{n}-q^{n-1} s_{0}\right),
$$

and hence, for a scalar $\lambda$ and sequences $x=\left(x_{n}\right), y=\left(y_{n}\right)$, we have: $N_{n}(x+y)=N_{n}(x)+N_{n}(y)$ and $N_{n}(\lambda x)=\lambda N_{n}(x)$.

In this paper, we study certain properties of a class of sequences (defined by using Euler transform) over an $n$-normed space using Musielak-Orlicz function. We introduce these spaces by using the Euler and some other matrix transformations. Finally, we present some inclusion relations between these spaces. Before proceeding further we present some definitions and results required for the further development of the article.

Definition 1.1. A continuous, convex, non-decreasing function $\varphi$ with $\varphi(x)>0$ for $x>0 ; \varphi(0)=0$ and $\varphi(x) \longrightarrow \infty$ whenever $x \longrightarrow \infty$ is called an "Orlicz function".

Orlicz sequence space [8], denoted $\ell_{\varphi}$ is space of sequences $x=\left(x_{n}\right)$ which satisfy $\sum_{k=1}^{\infty} \varphi\left(\frac{\left|x_{k}\right|}{t}\right)<\infty, t>0$. 
Lemma 1.2. [6] $\left(\ell_{\varphi},\|\|.\right)$ is a Banach space, where $\|x\|=\inf \{t>0$ : $\left.\sum_{j=1}^{\infty} \varphi\left(\frac{\left|x_{j}\right|}{t}\right) \leq 1\right\}$.

Definition 1.3. A "Musielak-Orlicz function" denoted by $\Phi$ is a sequence of Orlicz functions $\phi_{i}$.

For more information about the complementary function of the Musielak Orlicz function, Musielak Orlicz sequence space, Luxemburg norm we refer to $[8]$.

Remark 1.4. $\mathbf{N}, \mathbf{Z}, \mathbf{R}$ and $\mathbf{C}$ denotes set of natural numbers, integers, real numbers and complex numbers respectively.

Definition 1.5. A Lacunary sequence [3] is a sequence $\theta=\left(k_{r}\right)$ of positive integers with $k_{0}=0$, for $0<k_{r}<k_{r+1}$ and $h_{r}=\left(k_{r}-k_{r-1}\right) \rightarrow \infty$ as $r \rightarrow \infty$

Intervals $I_{r}=\left(k_{r-1}, k_{r}\right]$ are determined by $\theta$ and the ratio $\frac{k_{r}}{k_{r-1}}$ is denoted as $q_{r}$.

Another important notation that we require for this article is the notation of an $n$-norm.

Definition 1.6 ( $n$-norm, see $[\mathbf{5}, \mathbf{1 1}])$. A real valued function $\|\cdot, \cdots, \cdot\|$ defined on $V^{n}$, where $V$ is a linear space of dimension $d, d \geq n \geq 2, n \in \mathbf{N}$ over the (real or complex) field $\mathbf{K}$ is called an $n$-norm $([4,9])$ if the following conditions are satisfied:

1. $v_{1}, v_{2}, \cdots, v_{n}$ are linearly dependent iff $\left\|\left(v_{1}, v_{2}, \cdots, v_{n}\right)\right\|=0$;

2. $\left\|\left(v_{1}, v_{2}, \cdots, v_{n}\right)\right\|$ is invariant under permutations;

3. $\left\|\left(\alpha v_{1}, v_{2}, \cdots, v_{n}\right)\right\|=|\alpha|\left\|\left(v_{1}, v_{2}, \cdots, v_{n}\right)\right\| \forall \alpha \in \mathbf{K}$;

4. $\left\|\left(v+v^{\prime}, v_{2}, \cdots, v_{n}\right)\right\| \leq\left\|\left(v, v_{2}, \cdots, v_{n}\right)\right\|+\left\|\left(v^{\prime}, v_{2}, \cdots, v_{n}\right)\right\|$.

The pair $(V(\mathbf{K}),\|\cdot, \cdots, \cdot\|)$ is called an n-normed space.

Sequence spaces defined by Orlicz function have been introduced and their different properties have been investigated by Tripathy and Mahanta $[14,15]$. The notion of lacunary sequences have been investigated from different aspects by introducing different classes of lacunary spaces by Tripathy and Baruah [10], Tripathy and Dutta [12], Tripathy et al. [13], Tripathy and Sen [16] and others. For a detailed account of sequence spaces we refer the reader to $[1,2]$. 


\section{Main Results}

Let

$$
\mathbf{A}=\left[a_{i j}\right]=\left[\begin{array}{cccc}
p_{1} & w_{1}^{(1)} & w_{1}^{(2)} & \ldots \\
w_{1}^{(-1)} & p_{2} & w_{2}^{(1)} & \ldots \\
w_{1}^{(-2)} & w_{2}^{(-1)} & p_{3} & \ldots \\
\vdots & \vdots & \vdots & \vdots
\end{array}\right]
$$

where $p=\left(p_{i}\right)$ and $w^{(t)}=\left(w_{i}\right)^{(t)}$ are some fixed numerical sequences, $t \in \mathbf{Z} \backslash\{0\}$. For a fixed $k_{f} \in \mathbf{N}$ we define a finite sequence $t_{n}$ with $k_{f}$ terms by, $t_{n}=\left\{\begin{array}{ll}\frac{n+1}{2}, & \mathrm{n} \text { is odd; } \\ \frac{-n}{2}, & \mathrm{n} \text { is even. }\end{array}\right.$. We construct a matrix $\mathbf{A}_{\left(p, w^{t}, k_{f}\right)}=\mathbf{A}$, $w^{t_{i}}=0 \forall i>k_{f}$ and for $i=1,2, \ldots, k_{f}$ we have some fixed sequences $w^{t_{i}}$ and $p$.

Example 2.1. For $k_{f}=2$ we have $t_{1}=1, t_{2}=-1$, we define $p_{i}=-1 \forall i$ and $w_{i}^{(t)}=\left\{\begin{array}{ll}1, & \text { for } t=1,-1 \\ 0, & \forall t \in \mathbf{Z} \backslash\{0,1,-1\}\end{array} \quad\right.$ then we have,

$$
\mathbf{A}=\left[a_{i j}\right]=\left[\begin{array}{cccc}
-1 & 1 & 0 & \ldots \\
1 & -1 & 1 & \ldots \\
0 & 1 & -1 & \ldots \\
\vdots & \vdots & \vdots & \vdots
\end{array}\right]
$$

and hence, $\mathbf{A}_{\left(p, w^{t}, 2\right)} x=\left\langle\sum_{j=1}^{\infty} m_{i j} \xi_{j}\right\rangle_{n}=<-\xi_{1}+\xi_{2}, \xi_{1}-\xi_{2}+\xi_{3}, \xi_{2}-\xi_{3}+$ $\xi_{4}, \xi_{3}-\xi_{4}+\xi_{5} \ldots>$.

For a Musielak-Orlicz function $\Phi=\left(\varphi_{j}\right)$, we define the following sequence space:

$$
\begin{aligned}
& E_{n}^{q}\left(\Phi, u, p, s, \mathbf{A}_{\left(p, w^{t}, j_{f}\right)},\|\cdot, \cdots, \cdot\|\right)=\left\{x=\left(x_{j}\right): \lim _{r} \frac{1}{h_{r}} \sum_{j \in I_{r}} k^{-s}\right. \\
& \left.\left[\varphi_{j}\left(\left\|\left(\frac{u_{j} N_{j}\left(\mathbf{A}_{\left(p, w^{t}, j_{f}\right)}\right)}{t}, v_{1}, \cdots, v_{n-1}\right)\right\|\right)\right]^{p_{j}}<\infty, s \geq 0, \text { for some } t>0\right\} .
\end{aligned}
$$

Here, $p=\left(p_{j}\right)$ and $u=\left(u_{j}\right)$ are the bounded sequence of non-negative reals and sequence of positive reals; $h_{r}$ is as defined in definition 1.5; and $v_{i} \in V$.

Lemma 2.2 (Maddox, [7]). If $K=\max \left(1,2^{H-1}\right)$ and $0 \leq p_{j} \leq \sup p_{j}=$ $H$ then we have, $\left|a_{j}+b_{j}\right|^{p_{j}} \leq K\left\{\left|a_{j}\right|^{p_{j}}+\left|b_{j}\right|^{p_{j}}\right\}$ for all $j$ and $a_{j}, b_{j} \in \mathbf{C}$. Further we have, $|a|^{p_{j}} \leq \max \left(1,|a|^{H}\right)$ for all $a \in \mathbf{C}$. 
Theorem 2.3. $E_{n}^{q}\left(\Phi, u, p, s, \mathbf{A}_{\left(p, w^{t}, k_{f}\right)},\|\cdot, \cdots, \cdot\|\right)$ is a vector space over the field of complex numbers and is paranormed by the paranorm $g(x)$ defined by:

$$
\begin{gathered}
(x)=\inf \left\{t^{p_{n} / H}:\left(\operatorname { l i m } _ { r } \frac { 1 } { h _ { r } } \sum _ { k \in I _ { r } } \frac { 1 } { k ^ { s } } \left[\phi_{k}\right.\right.\right. \\
\left.\left.\left.\left\|\left(\frac{u_{k} N_{k}\left(\mathbf{A}_{\left(p, w^{t}, k_{f}\right)} x\right)}{t}, v_{1}, \cdots, v_{n-1}\right)\right\|\right]^{p_{k}}\right)^{\frac{1}{H}} \leq 1, n=1,2,3, \cdots\right\},
\end{gathered}
$$

$\max \left(1, \sup _{k} p_{k}\right)$.

Proof. Let, $x=\left(x_{k}\right)$ and $y=\left(y_{k}\right) \in E_{n}^{q}\left(\Phi, u, p, s, \mathbf{A}_{\left(p, w^{t}, k_{f}\right)},\|\cdot, \cdots, \cdot\|\right), \alpha, \beta \in$ C. There exist $t_{1}, t_{2} \in \mathbf{N}$ such that,

$$
\begin{gathered}
\lim _{r} \frac{1}{h_{r}} \sum_{k \in I_{r}} k^{-s}\left[\phi_{k}\left\|\left(\frac{u_{k} N_{k}\left(\mathbf{A}_{\left(p, w^{t}, k_{f}\right)} x\right)}{t_{1}}, v_{1}, \cdots, v_{n-1}\right)\right\|\right]^{p_{k}}<\infty \text { and } \\
\lim _{r} \frac{1}{h_{r}} \sum_{k \in I_{r}} k^{-s}\left[\phi_{k}\left\|\left(\frac{u_{k} N_{k}\left(\mathbf{A}_{\left(p, w^{t}, k_{f}\right)}\right)}{t_{2}}, v_{1}, \cdots, v_{n-1}\right)\right\|\right]^{p_{k}}<\infty .
\end{gathered}
$$

Since $\left(\phi_{k}\right)$ is a sequence of convex and non-decreasing functions. Let $t_{3}=\max \left(2|\alpha| t_{1}, 2|\beta| t_{2}\right)$ then we have,

$$
\begin{gathered}
\lim _{r} \frac{1}{h_{r}} \sum_{k \in I_{r}} k^{-s}\left[\phi_{k}\left\|\left(\frac{u_{k} N_{k}\left(\mathbf{A}_{\left(p, w^{t}, k_{f}\right)}(\alpha x+\beta y)\right)}{t_{3}}, v_{1}, \cdots, v_{n-1}\right)\right\|\right]^{p_{k}} \\
\lim _{r} \frac{1}{h_{r}} \sum_{k \in I_{r}} k^{-s}\left[\phi_{k}\left\|\left(\frac{u_{k} N_{k}\left(\mathbf{A}_{\left(p, w^{t}, k_{f}\right)} \alpha x\right)}{t_{3}}, v_{1}, \cdots, v_{n-1}\right)\right\|+\right. \\
\left.\left\|\left(\frac{u_{k} N_{k}\left(\mathbf{A}_{\left(p, w^{t}, k_{f}\right)}^{\beta y)}\right.}{t_{3}}, v_{1}, \cdots, v_{n-1}\right)\right\|\right]^{p_{k}} \\
K \lim _{r} \frac{1}{h_{r}} \sum_{k \in I_{r}} k^{-s}\left[\phi_{k}\left\|\left(\frac{u_{k} N_{k}\left(\mathbf{A}_{\left(p, w^{t}, k_{f}\right)}\right)}{t_{1}}, v_{1}, \cdots, v_{n-1}\right)\right\|\right]^{p_{k}}+ \\
K \lim _{r} \frac{1}{h_{r}} \sum_{k \in I_{r}} k^{-s}\left[\phi_{k}\left\|\left(\frac{u_{k} N_{k}\left(\mathbf{A}_{\left(p, w^{t}, k_{f}\right)}\right)}{t_{2}}, v_{1}, \cdots, v_{n-1}\right)\right\|\right]^{p_{k}}
\end{gathered}
$$

Therefore, $\alpha x+\beta y \in E_{n}^{q}\left(\Phi, u, p, s, \mathbf{A}_{\left(p, w^{t}, k_{f}\right)},\|\cdot, \cdots, \cdot\|\right)$. Hence, the space $E_{n}^{q}\left(\Phi, u, p, s, \mathbf{A}_{\left(p, w^{t}, k_{f}\right)},\|\cdot, \cdots, \cdot\|\right)$ is linear.

Further, we have, $g(x+y) \leq g(x)+g(y)$ and $g(x)=g(-x)$. Now, as $M_{k}(0)=0$, we have $\inf \left\{t^{p_{n} / \bar{H}}\right\}=0$ if $x=0$.

For any number $\lambda$,

$$
\begin{aligned}
& (\lambda x)=\inf \left\{t^{p_{n} / H}: \lim _{r} \frac{1}{h_{r}} \sum_{k \in I_{r}} k^{-s}\right. \\
& \left.\left[\phi_{k}\left\|\left(\frac{\lambda u_{k} N_{k}\left(\mathbf{A}_{\left(p, w^{t}, k_{f}\right)} x\right)}{t}, v_{1}, \cdots, v_{n-1}\right)\right\|\right]^{p_{k}} \leq 1, n=1,2,3, \cdots\right\}
\end{aligned}
$$

implies 


$$
\begin{gathered}
(\lambda x)=\inf \left\{(\lambda s)^{p_{n} / H}: \lim _{r} \frac{1}{h_{r}} \sum_{k \in I_{r}} k^{-s}\right. \\
\left.\left[\phi_{k}\left\|\left(\frac{u_{k} N_{k}\left(\mathbf{A}_{\left(p, w^{t}, k_{f}\right)}\right)}{s}, v_{1}, \cdots, v_{n-1}\right)\right\|\right]^{p_{k}} \leq 1, n=1,2,3, \cdots\right\},
\end{gathered}
$$

where, $s=\frac{t}{|\lambda|}$.

By Theorem 2.2 we get, $|\lambda|^{p_{k} / H} \leq\left(\max \left(|\lambda|^{H}, 1\right)\right)^{\frac{1}{H}}$ and this gives us,

$$
\begin{gathered}
(\lambda x) \leq\left(\max \left(|\lambda|^{H}, 1\right)\right)^{\frac{1}{H}} \inf \left\{(s)^{p_{n} / H}:\left(\lim _{r} \frac{1}{h_{r}} \sum_{k \in I_{r}} k^{-s}\right.\right. \\
\left.\left.\left[\phi_{k}\left\|\left(\frac{u_{k} N_{k}\left(\mathbf{A}_{\left(p, w^{t}, k_{f}\right)} x\right)}{t}, v_{1}, \cdots, v_{n-1}\right)\right\|\right]^{p_{k}}\right)^{1 / H} \leq 1 ; n=1,2, \cdots\right\} .
\end{gathered}
$$

Clearly, $g(x) \rightarrow 0$ when $x \rightarrow 0$ in $E_{n}^{q}\left(\Phi, u, p, s, \mathbf{A}_{\left(p, w^{t}, k_{f}\right)},\|\cdot, \cdots, \cdot\|\right)$.

Now let, $\lambda_{n} \rightarrow 0$ and $x \in E_{n}^{q}\left(\Phi, u, p, s, \mathbf{A}_{\left(p, w^{t}, k_{f}\right)},\|\cdot, \cdots, \cdot\|\right)$. For any $\epsilon>0$, let $n_{0} \in \mathbf{N}$ such that

$$
\lim _{r} \frac{1}{h_{r}} \sum_{k=n_{0}+1 \in I_{r}} k^{-s}\left[\phi_{k}\left\|\left(\frac{u_{k} N_{k}\left(\mathbf{A}_{\left(p, w^{t}, k_{f}\right)} x\right)}{t}, v_{1}, \cdots, v_{n-1}\right)\right\|\right]^{p_{k}}<\frac{\varepsilon}{2}
$$

for some $t>0$. This gives us

$$
\left(\lim _{r} \frac{1}{h_{r}} \sum_{k=n_{0}+1 \in I_{r}} k^{-s}\left[\phi_{k}\left\|\left(\frac{u_{k} N_{k}\left(\mathbf{A}_{\left(p, w^{t}, k_{f}\right)} x\right)}{t}, v_{1}, \cdots, v_{n-1}\right)\right\|\right]^{p_{k}}\right)^{\frac{1}{H}} \leq \frac{\varepsilon}{2} .
$$

Let $0<|\lambda|<1$, then by using the convexity of $\left(\phi_{k}\right)$, we have

$$
\begin{gathered}
\lim _{r} \frac{1}{h_{r}} \sum_{k=n_{0}+1 \in I_{r}} k^{-s}\left[\phi_{k}\left(\left\|\left(\frac{\lambda u_{k} N_{k}\left(\mathbf{A}_{\left(p, w^{t}, k_{f}\right)} x\right)}{t}, v_{1}, \cdots, v_{n-1}\right)\right\|\right]^{p_{k}}\right. \\
<|\lambda| \lim _{r} \frac{1}{h_{r}} \sum_{k=n_{0}+1 \in I_{r}} k^{-s}\left[\phi_{k}\left\|\left(\frac{u_{k} N_{k}\left(\mathbf{A}_{\left(p, w^{t}, k_{f}\right)} x\right)}{t}, v_{1}, \cdots, v_{n-1}\right)\right\|\right]^{p_{k}}<\left(\frac{\varepsilon}{2}\right)^{H} .
\end{gathered}
$$

Since $\left(\phi_{k}\right)$ is continuous everywhere on $[0, \infty)$, so

$$
h(t)=\lim _{r} \frac{1}{h_{r}} \sum_{k=1}^{n_{0}} k^{-s}\left[\phi_{k}\left\|\left(\frac{t u_{k} N_{k}\left(\mathbf{A}_{\left(p, w^{t}, k_{f}\right.}\right)}{t}, v_{1}, \cdots, v_{n-1}\right)\right\|\right]^{p_{k}}
$$

is also continuous at zero. Hence, there exists $0<\delta<1$ such that for some $0<t<\delta$ we have $|h(t)|<\varepsilon / 2$.

Let, $K(<n)$ be such that $\left|\lambda_{n}\right|<\delta$ then, we get

$$
\left(\lim _{r} \frac{1}{h_{r}} \sum_{k=1}^{n_{0}} k^{-s}\left[\phi_{k}\left\|\left(\frac{\lambda_{n} u_{k} N_{k}\left(\mathbf{A}_{\left(p, w^{t}, k_{f}\right)} x\right)}{t}, v_{1}, \cdots, v_{n-1}\right)\right\|\right]^{p_{k}}\right)^{\frac{1}{H}}<\frac{\varepsilon}{2} .
$$


Thus for $n>K$

$$
\left(\lim _{r} \frac{1}{h_{r}} \sum_{k \in I_{r}} k^{-s}\left[\phi_{k}\left\|\left(\frac{\lambda_{n} u_{k} N_{k}\left(\mathbf{A}_{\left(p, w^{t}, k_{f}\right)} x\right)}{t}, v_{1}, \cdots, v_{n-1}\right)\right\|\right]^{p_{k}}\right)^{\frac{1}{H}}<\varepsilon .
$$

Hence, $g(\lambda x)$ tends to 0 as $\lambda$ tends to 0 and hence the result.

Theorem 2.4. The space $E_{n}^{q}\left(\Phi, u, p, s, \mathbf{A}_{\left(p, w^{t}, k_{f}\right)},\|\cdot, \cdots, \cdot\|\right)$ is complete with paranorm $g(x)$ as defined in 2.3 .

Proof. The proof is routine verification, hence omitted.

Theorem 2.5. For "Musielak-Orlicz functions" $\Phi^{\prime}=\left(\phi_{k}^{\prime}\right)$ and $\Phi^{\prime \prime}=\left(\phi_{k}^{\prime \prime}\right)$ and for non-negative real numbers $s, s_{1}, s_{2}$, we have

(i) $E_{n}^{q}\left(\Phi^{\prime}, u, p, s, \mathbf{A}_{\left(p, w^{t}, k_{f}\right)},\|\cdot, \cdots, \cdot\|\right) \cap E_{n}^{q}\left(\Phi^{\prime \prime}, u, p, s, \mathbf{A}_{\left(p, w^{t}, k_{f}\right)},\|\cdot, \cdots, \cdot\|\right) \subseteq$ $E_{n}^{q}\left(\Phi^{\prime}+\Phi^{\prime \prime}, u, p, s, \mathbf{A}_{\left(p, w^{t}, k_{f}\right)},\|\cdot, \cdots, \cdot\|\right)$,

(ii) If $s_{2} \geq s_{1}$, then

$$
E_{n}^{q}\left(\Phi^{\prime}, u, p, s_{1}, \mathbf{A}_{\left(p, w^{t}, k_{f}\right)},\|\cdot, \cdots, \cdot\|\right) \subseteq E_{n}^{q}\left(\Phi^{\prime}, u, p, s_{2}, \mathbf{A}_{\left(p, w^{t}, k_{f}\right)},\|\cdot, \cdots, \cdot\|\right),
$$

(iii) If $\Phi^{\prime}$ and $\Phi^{\prime \prime}$ are equivalent, then

$E_{n}^{q}\left(\Phi^{\prime}, u, p, s, \mathbf{A}_{\left(p, w^{t}, k_{f}\right)},\|\cdot, \cdots, \cdot\|\right)=E_{n}^{q}\left(\Phi^{\prime \prime}, u, p, s, \mathbf{A}_{\left(p, w^{t}, k_{f}\right)},\|\cdot, \cdots, \cdot\|\right)$.

Proof. The proof of the theorem can be established using standard techniques, so omitted.

Theorem 2.6. $\begin{gathered}{ }_{n}^{q}\left(\Phi, u, r, s, \mathbf{A}_{\left(p, w^{t}, k_{f}\right)},\|\cdot, \cdots, \cdot\|\right) \subseteq E_{n}^{q}\left(\Phi, u, p, s, \mathbf{A}_{\left(p, w^{t}, k_{f}\right)},\right. \\ \|\cdot, \cdots, \cdot\|), 0<r_{k} \leq p_{k}<\infty .\end{gathered}$

Proof. Let, $x \in E_{n}^{q}\left(\Phi, u, r, s, \mathbf{A}_{\left(p, w^{t}, k_{f}\right)},\|\cdot, \cdots, \cdot\|\right)$. Then, there exist some $t>0$ such that

$$
\lim _{r} \frac{1}{h_{r}} \sum_{k \in I_{r}} k^{-s}\left[\phi_{k}\left\|\left(\frac{u_{k} N_{k}\left(\mathbf{A}_{\left(p, w^{t}, k_{f}\right)} x\right)}{t}, v_{1}, \cdots, v_{n-1}\right)\right\|\right]^{r_{k}}<\infty .
$$

Hence, $\phi_{k}\left\|\left(\frac{u_{k} N_{k}\left(\mathbf{A}_{\left(p, w^{t}, k_{f}\right)} x\right)}{t}, v_{1}, \cdots, v_{n-1}\right)\right\| \leq 1$ for sufficiently large $k$. For some fixed $k_{0} \in \mathbf{N}$ let, $k \geq k_{0}$. As $\left(M_{k}\right)$ is non-decreasing so

$\lim _{r} \frac{1}{h_{r}} \sum_{k \geq k_{0} \in I_{r}} k^{-s}\left[\phi_{k}\left\|\left(\frac{u_{k} N_{k}\left(\mathbf{A}_{\left(p, w^{t}, k_{f}\right)} x\right)}{t}, v_{1}, \cdots, v_{n-1}\right)\right\|\right]^{p_{k}}$

$\leq \lim _{r} \frac{1}{h_{r}} \sum_{k \geq k_{0} \in I_{r}} k^{-s}\left[\phi_{k}\left\|\left(\frac{u_{k} N_{k}\left(\mathbf{A}_{\left(p, w^{t}, k_{f}\right)} x\right)}{t}, v_{1}, \cdots, v_{n-1}\right)\right\|\right]^{r_{k}}<\infty$.

Hence, $x \in E_{n}^{q}\left(\Phi, u, p, s, \mathbf{A}_{\left(p, w^{t}, k_{f}\right)},\|\cdot, \cdots, \cdot\|\right)$.

We state the following result without proof in view of Theorem 2.6. 
Theorem 2.7. (i) If $0<p_{k} \leq 1$, then we have

$E_{n}^{q}\left(\Phi, u, p, s, \mathbf{A}_{\left(p, w^{t}, k_{f}\right)},\|\cdot, \cdots, \cdot\|\right) \subseteq E_{n}^{q}\left(\Phi, u, s, \mathbf{A}_{\left(p, w^{t}, k_{f}\right)},\|\cdot, \cdots, \cdot\|\right)$.

(ii) If $p_{k} \geq 1$ for every natural number $k$, then

$E_{n}^{q}\left(\Phi, u, s, \mathbf{A}_{\left(p, w^{t}, k_{f}\right)},\|\cdot, \cdots, \cdot\|\right) \subseteq E_{n}^{q}\left(\Phi, u, p, s, \mathbf{A}_{\left(p, w^{t}, k_{f}\right)},\|\cdot, \cdots, \cdot\|\right)$.

\section{Conclusion}

We have defined and studied the properties of certain new Lacunary sequence spaces defined over an $n$-normed space. The new space is defined by using a combination of matrix and Euler summability transformation methods. Some inclusion relations between these spaces are proved.

\section{References}

[1] N. L. Braha and M. Et, "The sequence space $E_{n}^{q}(M, p, s)$ and $N_{k}-$ lacunary statistical convergence", Banach Journal of Mathematical Analysis, vol. 7, pp. 88-96, 2013.

[2] R. Colak, B. C. Tripathy and M. Et, "Lacunary strongly summable sequences and q-Lacunary almost statistical convergence", Vietnam Journal of Mathematics, vol. 34, pp. 129-138, 2006.

[3] A. R. Freedman, J. J. Sember, and M. Raphael, "Some Cesaro-type summability spaces", Proceedings of the London Mathematical Society, vol. 3, pp. 508-520, 1978.

[4] S. Gähler, "Lineare 2-normierte Rume", Mathematische Nachrichten, vol. 28, pp. 1-43, 1964.

[5] H. Gunawan and M. Mashadi, "On n-normed spaces", International Journal of Mathematics and Mathematical Sciences, vol. 27, pp. 631-639, 2001.

[6] J. Lindenstrauss and L. Tzafriri, "On Orlicz sequence spaces", Israel Journal of Mathematics, vol. 10, pp. 379-390, 1971.

[7] I. Maddox, Elements of functional analysis. Cambridge: Cambridge University Press, 1988.

[8] J. Musielak, Orlicz spaces and modular spaces. Berlin: Springer, 2006. 
[9] A. Misiak, "n-inner product spaces", Mathematische Nachrichten, vol. 140, pp. 299- 319, 1989.

[10] B. C. Tripathy and A. Baruah, "Lacunary statistically convergent and lacunary strongly convergent generalized difference sequences of fuzzy real numbers", Kyungpook Mathematical Journal, vol. 50, no. 4, pp. 565-574, 2010.

[11] B. C. Tripathy and S. Borgogain, "On a class of n-normed sequences related to the $\ell^{p}$-space", Boletim da Sociedade Paranaense de Matemática, vol. 31, no. 1, pp. 167-173, 2013.

[12] B. C. Tripathy and A.J. Dutta, "Lacunary bounded variation sequence of fuzzy real numbers”, Journal of Intelligent \& Fuzzy Systems, vol. 24, no. 1, pp. 185-189, 2013.

[13] B. C. Tripathy, B. Hazarika and B. Choudhary, "Lacunary I-convergent sequences”, Kyungpook Mathematical Journal, vol. 52, no. 4, pp. 473-482, 2012.

[14] B. C. Tripathy and S. Mahanta, "On a class of generalized lacunary difference sequence spaces defined by Orlicz function", Acta Mathematicae Applicatae Sinica, English Series, vol. 20, no. 2, pp. 231-238, 2004.

[15] B. C. Tripathy and S. Mahanta, "On a class of difference sequences related to the $\ell_{p}$ space defined by Orlicz functions", Mathematica Slovaca, vol. 57, no. 2, pp. 171-178, 2007.

[16] B. C. Tripathy and M. Sen, "On lacunary strongly almost convergent double sequences of fuzzy numbers", Analele Universitatii din Craiova. Seria Matematica-Informatica, vol. 42, no. 2, pp. 254-259, 2015.

\author{
Pranav Sharma \\ School of Basic Sciences, \\ IIMT University, \\ Meerut, Uttar Pradesh 250001 \\ India \\ e-mail: pranav15851@gmail.com
}

